\title{
L'imaginaire animal
}

\author{
Peter Berz, Berlin
}

R S I - Real Symbolisch Imaginär: diese Dreifaltigkeit, so der Psychoanalytiker und Epistemologe Jacques Lacan, artikuliere das menschliche Subjekt. Nicht nur in „der Erfahrung“, also den Reden einer von Freud installierten Praxis, in der die Geschichte des Wahnsinns auf jenes einzige Paar zuläuft: den Wahnsinnigen und den Arzt. ${ }^{1}$ Vielmehr existiert Lacans dreifaltiges Subjekt als eine Geschichte des Wissens: von Physik, physikalischer Optik, experimenteller Gestaltpsychologie, von Mathematik, Nachrichtentheorie, Philosophie und schließlich, in einer psychoanalytischen Tradition seit Freud, von biologischem Wissen.

Den Term des Imaginären hat Ulrike Kadi schlicht als „Bilderwahn“ angesprochen und wissens- wie kulturhistorisch situiert. ${ }^{2}$ Seit seinen Anfängen operiert der junge Psychiater der Polizeipräfektur von Sainte-Anne mit Bildern. Schon im Buch über die Psychose Marguerite Pantins von 1932 steht an allen Knotenpunkten des Wahns ein Bild. Es ist die bekannte Film- und Theater-Schauspielerin Huguette Duflos, die Marguerite alias Aimée schließlich als Feindin heimsucht, bevor sie dieser eines Tages mit einem Messer zusetzt. Aimée begegnet den Filmplakaten ihrer Feindin - so Lacan ausdrücklich - permanent auf den Straßen von Paris. Zwischen Bildern und Gerüchten, geflüstert auf dem Treppenabsatz, im Flur, auf der Straße entstehe der Wahn. Hier beginne jene „Reihe von Identifikationen“ oder Bild-Ersetzungen, die Lacan als Psychose analysiert, bis in die Phantastik von Aimées Romanen. ${ }^{3}$ In der Arbeit des jungen Psychiaters, die vom bildsüchtigen Salvador Dalí ohne Verzögerung aufgenommen wird ${ }^{4}$, taucht die Psychoanalyse nur am Horizont auf. Bestimmung der Lage: mitten in der Psychoanalyse liege eine terra incognita, „mythisch und unbekannt“, und die heißt Narzißmus. ${ }^{5}$ Die Liebe von Narziß und der Nymphe Echo entfaltet in der Ovid'schen 
Erzählung die dramatische Geschichte einer Bildfaszination. Lacan, der den Narzißmus 1938 ,aus dem Vollsinn des Narziß-Mythos ${ }^{\text {“6 }}$ verstehen möchte, entwickelt daraus 1936/1949 die bekannte Theorie vom Bild des eigenen Körpers als Epizentrum aller Ichbildung. ${ }^{7}$ Es liegt programmatisch neben Freud. Denn alles hätte Freud gesehen, nur eins sei seinem Assoziationismus, Stand der Psychologie Ende des 19. Jahrhunderts, entgangen: das Bild, die Gestalt. ${ }^{8}$ Von diesem Ausgangspunkt her, der sich wissensgeschichtlich in der gestaltpsychologisch und phänomenologisch orientierten Psychiatrie verorten läßt (mit ihr sind Lacans Anfänge nicht weniger eng verbunden als die Foucaults), unternimmt Lacan seine Relektüre Freuds.

Freilich entging es ihm als einem der ersten Leser von Freuds erst 1950 erschienen „Entwurf einer Psychologie“ (1895) nicht, daß ein turning point im Freudschen Denken der Übergang von der Besetzung einzelner Neuronen zur Besetzung von „Neuronen-Komplexen“ und schließlich „Erinnerungsbildern“ ist. ${ }^{9}$ Die Bilder der Psychoanalyse sind besetzbar, in einer Art „Metabolismus der Bilder ${ }^{\text {"10 }}$, und darum pathologisch fixierbar. ${ }^{11}$ Sie stehen in einer großen, noch ungeschriebenen Geschichte: der Geschichte der Faszination, nicht nur in der Geschichte ikonologischen Deutens und Bedeutens. Sprechend erst, im Prozeß verschiebbarer Adressen und Zeichen, zehren sich die Bilder auf.

Was das Folgende nun zu denken versucht, ist die einfache Tatsache, daß überall da, wo Lacan - von den 30ern über die 50er bis in die 60er Jahre - vom Imaginären spricht, eine Reihe von Wesen Pate stehen, die wir als Tiere ansprechen. Die Tiere, so die Behauptung, leben im Imaginären: „l'imaginaire est assurément guide de vie pour tout le champ animal“. ${ }^{12}$ Die Tiere leben in und mit Bildern, im Zeigen, zu sehen Geben, Bild Machen, ob verbergend oder spektakulär, exzessiv erscheinend..$^{13}$ Animals in mission ${ }^{14}$ : bei Lacan ist die Mission der Tiere das Bild.

Das ist einigermaßen erstaunlich. Denn auf dem state of the art biologischen Denkens, für das im Kampf der Arten fitness die einzige biologische Realität ist, leben die Tiere eine umfassende, alles durchdringende economy of nature, die nicht erst durch die Molekularbiologie eine technische geworden ist. Und doch entscheidet sich die Biologie des Verhaltens in einem bestimmten Moment ihrer Geschichte an der Frage nach dem Bild, seinen imaginären Übertreibungen und illusionären Überschüssen. Von dort wird die Ethologie des Bildes im Denken Lacans ankommen. 


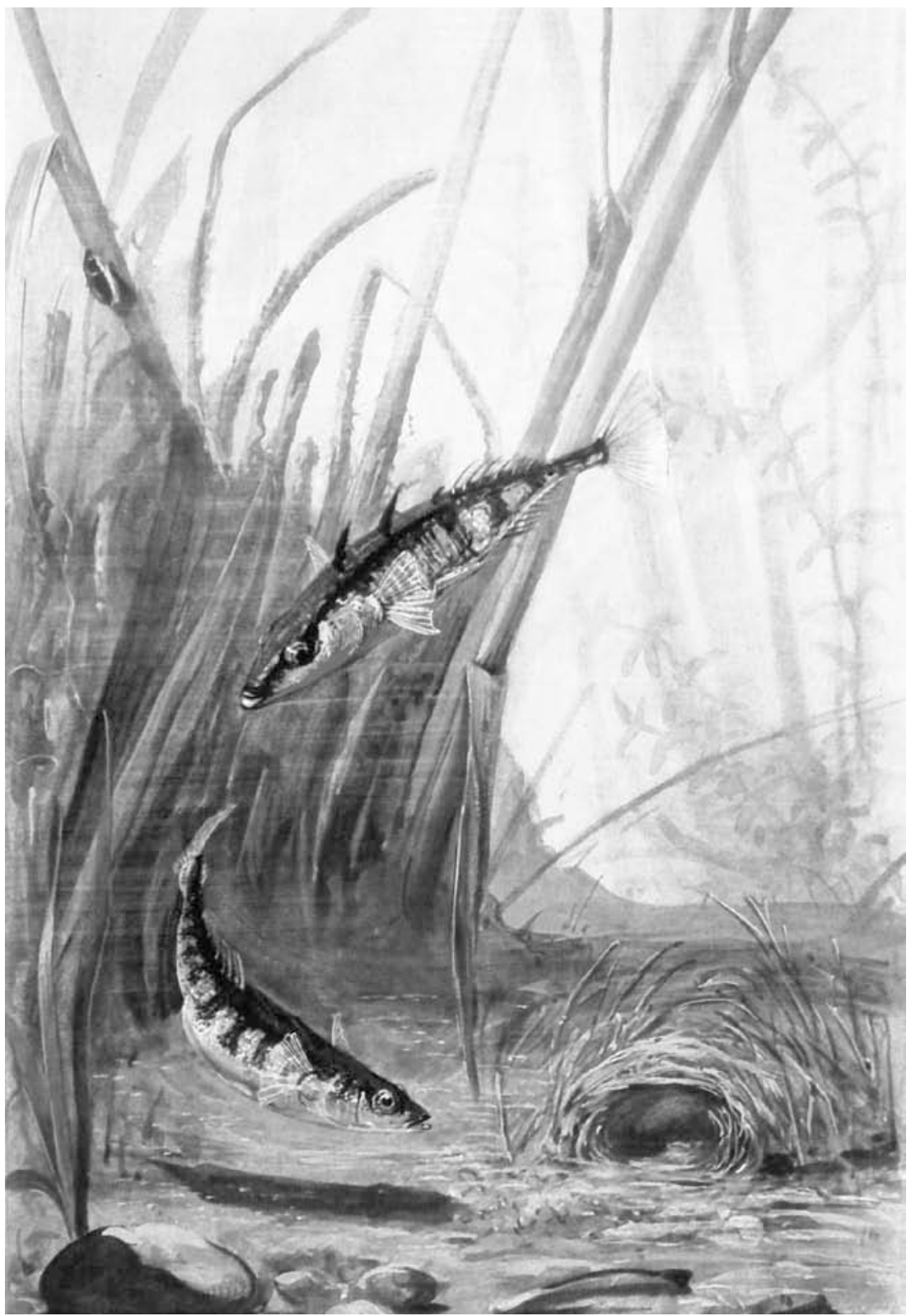

Abb. 1 Stichlingspärchen Brehms Tierleben (1914) 


\section{Der Stichling}

Ich beginne die Frage nach dem Imaginären der Tiere im Wasser. Mit einem ebenso urtümlichen wie ökonomisch nutzlosen Tier: dem dreistachligen Stichling, Gasterosteus aculeatus Linné, der sich fast überall in Europa findet außer, leider, im Donauraum. (Urtümlich nennt man ihn, weil dieser Fisch noch gar keine Schuppen hat, sondern je nach Art größere oder kleinere Panzerplatten.) Nicolaas Tinbergen, zusammen mit Konrad Lorenz erster Nobelpreisträger für Verhaltensbiologie, hat zusammen mit dem Stichling 1936 seine Wissenschaft begründet.

Das Bild Machen des Stichlings setzt spektakulär vor allem im Frühjahr ein. „Der Fisch errötet, überholt den Schwarm“, heißt es bei Ingeborg Bachmann, einer Donauraumbewohnerin und vermutlich Leserin von Annie Francé-Harrars wunderlichem Büchlein „Tier und Liebe“. ${ }^{15}$ Beim Männchen des Stichlings rötet sich der Bauch (vgl. Abb. 1). Dieses Rot ist eine Drohung für Konkurrenten. Wird es aber durch ein leichtes, irisierendes Blau auf dem Rücken ergänzt, dann verführt und lockt der rote Bauch das Weibchen. Das Rot leitet eine geschlossene Kette von Balzhandlungen ein: von Momenten, Bewegungen, stills des Bild Machens. Immer antwortet eine Bewegung des Männchens auf eine des Weibchens (vgl. Abb. 2). So führt das Männchen das Weibchen in ein Nest, das es zuvor im Sand gegraben und aus Stengeln gebaut hat. Auf den einen Tanz des Männchens im Zickzack folgt eine Bewegung des Weibchens: es stellt sich auf und zeigt frontal den breiten Bauch mit den Eiern (vgl. Abb. 3). Das Männchen schwimmt daraufhin in Richtung zum Nest, es führt, das Weibchen folgt (vgl. Abb. 1); das Männchen legt sich flach auf die Seite vors Nest; das Weibchen kriecht in das tunnelartige Nest; das Männchen beginnt - über dem Weibchen stehend - heftig zu zittern.

Zur Balzzeit stecken die Männchen aber auch Reviere ab. Ein fremder Stichling mit roter Unterseite im eigenen Revier wird sofort, oft tödlich, mit den Stacheln und dem Maul angegriffen. In bestimmten Situationen, über die noch zu sprechen sein wird, tritt ein Moment der Parade ein, eine Drohhaltung: der Fisch stellt sich senkrecht, mit dem Mund nach unten, dabei oft im Sand grabend (vgl. Abb. 4). Er streckt währenddessen genau die Flosse und den Stachel, die zum Gegner zeigen, weit von sich weg.

Das alles sprechen Verhaltensbiologen wie Lorenz und Tinbergen seit 1935 als eine Kaskade von Auslösern an, sogenannten releasers oder déclencheurs. Sie lösen bestimmte „Triebhandlungen“ aus. Die Auslöser im vorliegenden 


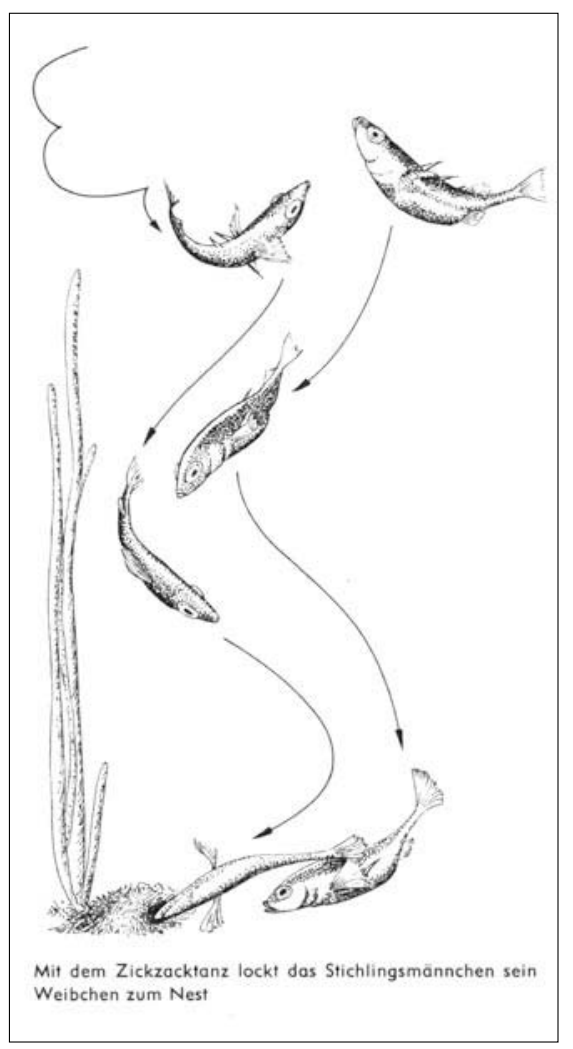

Abb. 2

Zickzacktanz als Balzverhalten des männlichen Stichlings.

Urania Tierreich (1967)

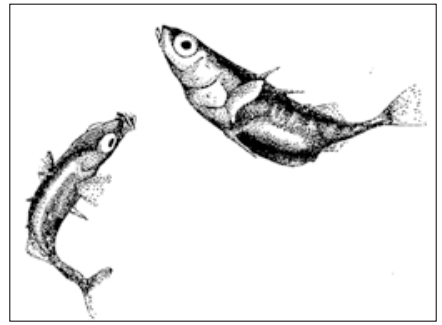

Abb. 3

Balzendes Stichlingspärchen, links das Männchen beim „Zickzacktanz", rechts balzendes Weibchen.

Zeitschrift für Tierpsychologie (1937)

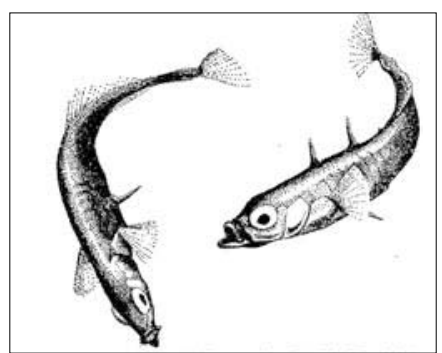

Abb. 4

Kämpfende Stichlingsmännchen. Das linke Tier "droht".

Zeitschrift für Tierpsychologie (1937)

Fall operieren im Optischen. Als eine Bewegungsform, eine Geste oder die Struktur einer ruhenden Gestalt, einer Konfiguration. Tinbergens erste Experimente zeigten, daß jedes beliebige Objekt beim Stichling die Kampfhandlungen auslöst, es kann rund, eckig, fischförmig sein, es muß nur der einen Bedingung genügen, daß die untere Partie rot ist. Die Form spielt keine Rolle, was zählt, ist eine minimale Konfiguration: unten rot, oben nicht rot. Wo aber ist im Auslöser dann ein Bild? 
Vier sehr grobe Antworten aus Konrad Lorenz' epochaler Arbeit über den „Kumpan in der Umwelt des Vogels“ von 1935.

\section{Das Bild im Auslöser}

Lorenz versetzt die Frage nach dem Bild aus dem Wasser an die Luft. Das ist: zu den, nach Hegel, theoretischsten aller Tiere, den Vögeln. Denn „,die Vögel schweben frei in der Luft, als ihrem Elemente; von der objektiven Schwere der Erde getrennt, erfüllen sie die Luft mit sich und äußern ihr Selbstgefühl im besonderen Elemente“, also singen. ${ }^{16}$

(Aber schon das Wissen von den Tieren, nicht erst ihr Sein, wäre bis in letzte epistemologische Feinheiten von ihrem jeweiligen Element, Milieu oder „Medium“ zu denken. Entweder dem Wasser, die Fische verbracht ins Aquarium als geschlossenes Milieu und Schema einer Laborlage, in der, wie bei Tinbergen, alle Ereignisse bildschön hinter Glas stattfinden. Oder von der Luft her, die Vögel verbracht in offene Käfige oder auf Dächer, Tier und Forscher pendelnd zwischen festem Boden, Sumpf, Baum, Luftraum, wie Lorenz die lustvollsten Momente seines Forscherdaseins beschreibt. ${ }^{17}$ )

\section{Ding Merkmal Schema}

Theoretisch beginnt 1935 nicht nur im Altenberger Sommer, sondern auch im Freiburger Wintersemester alles mit der „Frage nach dem Ding“ ${ }^{18}{ }^{18}$ Wo bei Heidegger Ding und Gegenstand in der neuzeitlichen Naturwissenschaft als Ganzer auf dem Spiel stehen, da konstatiert Lorenz kurz: das Ding ist biologisch gesehen überhaupt nicht notwendig. Ding- oder Gegenstandserfassen begreift Lorenz gestaltpsychologisch, von der Konstanz des Dings her, so wie etwa die Psychologen Gelb und Goldstein die „Farbkonstanz der Sehdinge“: physikalisch ist im Abendlicht alles anders als bei Tag, trotzdem bleibt die Orange orange; ein Rechteck, von der Seite gesehen, bleibt ein Rechteck. All das läßt sich psychophysiologisch vom Tintenfisch bis zum Menschen sehr gut als „Wiedererkennen“ durchexperimentieren.

Nun leben aber die meisten Lebewesen ganz ohne ein Ding Erfassen ihrer Umwelt. Ein Merkmal, roter Bauch oder aufgerichtete Flosse, genügt, um die biologisch notwendigen Handlungsketten in Gang zu setzen. „Ding“ könnte allenfalls eine zufällige, nur biologisch wahrscheinliche Zusammenkunft 
verschiedener Auslöser heißen. Denn die Auslöser lassen sich leicht wieder voneinander trennen, wenn etwa eines evolutiven Tages und sehr unwahrscheinlich ein systematisch experimentierendes Wissenstier auftritt: der Biologe. Durch einfache Attrappen kann er jeden Auslöser ersetzen und so die Auslöser isolieren.

Biologisch gibt es allerdings ein Problem mit den einfachen Auslösern. Sachen, die unten rot sind, gibt es viele. Das heißt: die Gefahr der Verwechslung oder Täuschung ist sehr hoch - mit Folgen auf Leben und Tod. Nur möglichst unwahrscheinliche Merkmale sind gegen Verwechslung gefeit und damit gute Merkmale. Unwahrscheinlichkeit ist eine biologische Notwendigkeit. Nun ist aber ein einzelnes Merkmal im Gewimmel der Erscheinungen nicht so leicht unwahrscheinlich zu machen wie zwei oder mehrere Merkmale oder eine ganze Konstellation von Merkmalen. Lorenz nennt eine solche Konstellation, Uexküll folgend, „Schema“.${ }^{19}$ Wohl als erster Biologe bringt er in der Kumpan-Arbeit, geschrieben zu Ehren Uexkülls, die Wahrscheinlichkeitsfrage ins Spiel, also ein kommunikationstheoretisches Dispositiv avant la lettre de Claude Shannon. ${ }^{20}$

Lorenz' Kommunikationstheorie stammt, wie Veronika Hofer gezeigt hat, aus der Proto-Kybernetik seines Lehrers, des Wiener Psychologen Karl Bühler. ${ }^{21}$ Die Krise der Psychologie (Lacan wird sie von Neuem ausrufen) beginnt bei Bühler 1929 mit dem tierischen Verhalten. Alles an ihm sei „Kommunikationsmittel“. ${ }^{22}$ Bis hin zur menschlichen Sprache entfalte es sich in drei Dimensionen: Kundgabe, Auslösung, Darstellung. Darwins Theorie der Ausdrucksbewegung, Expression of Emotions (1872), habe übersehen, daß zu jedem Kundgeber ein Kundnehmer gehöre, alles also mit dem Adressaten der Bewegung anfange, mit „zwei Mitgliedern einer Gemeinschaft“ und nicht mit dem Ausdruck innerer Zustände oder Stimmungen. ${ }^{23}$ Am reinsten sieht Bühler das „Zweiersystem von Sender und Empfänger“, diesen „Quellpunkt der Sprache ${ }^{\text {"24 }}$ in technischen Dispositiven verkörpert, die er als solche erst gar nicht zu nennen wagt: Fernschreiber, Telegraph, Telephon. ${ }^{25}$ Denn Kundgabe sei immer auch Auslösung von anderem Verhalten. ${ }^{26}$ Dabei empfehle sich weniger der Begriff des „Verstehens“ als der der „Steuerung“. ${ }^{27}$ Gut kybernetisch beginnt sie nicht mit einem einzigen Chauffeur, der einem blind dahintrottenden Passanten ausweicht, sondern dann, wenn ein Chauffeur einem anderen Chauffeur ausweicht, also mit ,gegenseitiger Steuerung““.$^{28}$ Nur hier entstehe im Verhalten Bedeutung, also das „sinnvolle Benehmen“ der Mitglieder einer „echten Gemeinschaft“, die auch für das Tierreich anzunehmen ist (und nicht 
„Zufallsscharungen von Einsiedlern“).${ }^{29}$ Die materiale Bedingung aber für das Entstehen sinnvollen Benehmens ist, daß es sich als „,abzählbare Menge wohl charakterisierter Elemente und Komplexe" vom Rauschen der Ereignisse unterscheidet, also „sich aus dem unübersehbaren Reich sinnloser Möglichkeiten das System des sinnvollen Benehmens heraushebt" ${ }^{30}$ Shannons Nachrichtentheorie würde von Filtern sprechen.$^{31}$ Diese theoretische Herkunft von Lorenz' Fragen, die seinem Kollegen Tinbergen etwa ganz abgeht, macht es verständlicher, warum Auslöser und Bild der Lorenzschen Verhaltensbiologie nach dem zweiten Weltkrieg mühelos in eine „kybernetisch psychologische Theorie" einzuführen sein werden. ${ }^{32}$ (Daß der dritte, sprachphilosophische Term, die Darstellung, in Bühlers radikaler Kommunikationstheorie nicht mit der Beschreibung von Tatsachen und deren Logik beginnt, sondern dort, wo bei Lacan das Register des Symbolischen anfängt: mit Lüge und Recht, la parole menteresse, les lois et les contrats, dies sei nur nebenbei erwähnt. ${ }^{33}$ )

\section{Unwahrscheinlich}

Wahrscheinlich oder unwahrscheinlich, das ist für Lorenz auch der Ansatzpunkt, eine Evolution biologischer Schemata zu denken. ${ }^{34}$ Sie beginnt mit der fundamentalen Unterscheidung von belebt und unbelebt. Ein unbelebtes Ding, ein Halm zum Nestbau etwa, hat nur wenige Merkmale. Seine generelle Unwahrscheinlichkeit läßt sich darum kaum über ein bestimmtes Maß steigern. Dagegen steckt in einem Artgenossen ein ungeheurer Detailreichtum. Da nun der Artgenosse als Auslöser und der Adressat des Auslösers beide veränderlich sind und beide Teil der gleichen Evolution, können sich die Schemata ihrer Kommunikation leicht in Richtung Unwahrscheinlichkeit differenzieren.

Was dabei entsteht, sind Zeichen. Ein Zeichen ist nach Lorenz das Unwahrscheinliche. Zeichenhafte Bewegungen etwa können sich aus sogenannten „Intentionsbewegungen“ entwickeln: wenn die Entenmutter ihren Kücken vorausläuft und einmal von ihrem geraden Weg abbiegt, macht sie vorher eine forcierte, schnelle und intensive Nickbewegung in die Abbiege-Richtung. Eine solche Intentionsbewegung kann sich „formalisieren“ oder „ritualisieren“ und wird dann zum Zeichen. Denn wo es nur darauf ankommt, daß die Kommunikation zwischen Artgenossen funktioniert, da kann sich die Intentions-Bewegung durch ,mimische Übertreibung“ schnell von jeglichem Signifkat lösen, indem etwa Intentionsbewegungen „,bis zum Grotesken un- 
terstrichen und überbetont" werden oder auch durch übertriebene Farboder Formmerkmale ergänzt. ${ }^{35}$ Dann fangen sie an zu bedeuten.

Die Entwicklung zu immer höherer Unwahrscheinlichkeit kennt keine äußeren Grenzen, weil es ja nicht um Signifkate, sondern nur um intra-spezifische Kommunikation geht. Lorenz wird es in den kybernetischen 50er Jahren nachrichtentheoretisch reformulieren: „Wo ein intra-spezifisches System von Signal-aussendenden und Signal-empfangenden Differenzierungen ausgebildet wurde, dort ist die Form der Signale so gut wie ausschließlich historisch durch die ,Konvention` zwischen Reizsender und Reizempfänger bestimmt und hat nur mehr sehr lose Beziehungen zur Außenwelt. ${ }^{“ 36}$ Wegen dieser Lösung von der Außenwelt lassen sich die Schemata zu einer „beliebig hohen generellen Unwahrscheinlichkeit" treiben $^{37}$, bis zu einer oft irrwitzigen Spezialisierung, ohne prinzipielle Außenweltgrenzen. Der Pfau ist „unwahrscheinlich schön": denn er ist eben überhaupt unwahrscheinlich.

\section{Komplexqualität}

An wahrscheinlich/unwahrscheinlich, einfach/kompliziert als Grundparametern einer Lorenzschen Bildtheorie entscheidet sich zuletzt auch die große, biologisch brisante Frage nach ererbt oder erworben.

Die Auslöser-Schemata sind einfach, weil ererbt, und ererbt, weil einfach. Das ist Lorenz' Urszene: daß die Auslöser einfach sind und sich darum einfach experimentell erzeugen lassen, durch, wie erwähnt, das Hauptinstrument früher Verhaltensforschung, die Attrappe. Mit ihr läßt sich ein einziges Auslösermerkmal simulieren, isolieren oder auch ins Übernormale steigern. Angeborene „Urbilder“, alias Jungsche Archetypen, wären nach Lorenz schon darum ein Unsinn. Das Angeborene ist einfaches Merkmal, aber weder Bild noch Schema.

Dagegen sind erworbene Schemata, erworben durch Dressur oder schließlich Prägung, ihrem Wesen nach kompliziert. In ihnen sind, so Lorenz, ,alle Merkmale einer Situation zu einer Komplexqualität vereinigt" ${ }^{38}$ Kein Teil des Schemas kann vom anderen getrennt werden. Das gilt vor allem für die „Eigendressuren“. Wenn in einer bestimmten Situation eine bestimmte Bewegung mit dem Halm fürs Nest zum Erfolg führte, wird der Vogel diese Bewegung „nur in einer in allen Einzelheiten gleichen [Gesamt-]Situation“ wiederholen. Denn woher soll er einzelne, charakteristische Merkmale unterscheiden, es sei denn im unwahrscheinlichen Fall, daß in verschiedenen Situa- 
tionen immer ein einziges Merkmal gleich bleibt und alle anderen variieren? ${ }^{39}$ Lorenz spricht die „Komplexqualität“ oder „Komplexgestalt“ eines Auslösers als „Gesamtbild“ oder schlicht „Bild“ an. ${ }^{40}$

Vor allem jener besondere Typ von Erwerbung, der Lorenz berühmt machte, die „Prägung“, handelt durchweg von solchen Komplexgestalten oder Bildern. Schon Heinroth schrieb (so zitiert Lorenz), daß die junge Graugans das erste Wesen, auf das sie trifft, ,wirklich in der Absicht ansieht, sich das Bild genau einzuprägen“. ${ }^{41}$ Aus Merkmal und Schema wird „das Bild des Elterntiers“ “42

Freilich gibt es keine Prägung ohne daß sie mit ererbten, einfachen Schemata zusammenspielte. Vielmehr vereinigt die Prägung „die einzelnen, von einander unabhängigen [ererbten, PB] Auslöse-Schemata einzelner Triebhandlungen, wie ein Ausnähbild für Kinder sich zwischen die vorgestochenen Löcher einfügt, die an sich keine Beziehungen zueinander haben und nur durch einen Funktionsplan zusammengefaßt werden, dessen Ganzheitlichkeit von einer andern Seite her bestimmt wird. " 43 Außerdem haben die geprägten Bilder mit den ererbten Merkmalen oder Schemata gemeinsam, daß sie unwiderruflich sind. Sie können nicht wie andressierte Bilder wieder vergessen werden. ${ }^{44}$ Die geprägten Bilder bekommen bei Lorenz den gleichen Status wie die Organe des Organismus. Bilder als Organe: Lorenz beruft sich dafür emphatisch auf eine nobelpreisverdächtige Wissenschaft, die Entwicklungsbiologie Hans Spemanns. ${ }^{45}$ Der Basler Biologe Adolf Portmann, der wie kein zweiter die tierische Erscheinung in den Mittelpunkt seines Denkens stellte, wird dereinst die gesamte sichtbare Außenseite der Tiere, das, was sie zeigen und zu sehen geben, als ein eigenständiges Organ bezeichnen, von gleicher funktioneller und struktureller Komplexität wie Herz und Auge. ${ }^{46}$

\section{Objekt Bild}

Schließlich aber kommt mit den geprägten Bildern bei Lorenz etwas in den Blick, was seinem Denken unvermutete Anschlußmöglichkeit schaffen wird: die „objektlos ererbten Triebhandlungen “ ${ }^{47}$ So ist etwa das Nachlaufen, diese komplizierte Handlungsfolge der jungen Kücken, zwar genauestens ererbt. Aber es hat zunächst kein „Objekt“. Auch wenn in Lorenz' spontaner Philosophie des Wissenschaftlers die Begriffe von Ding und Gegenstand und Objekt oft durcheinanderwirbeln, stellt sich die prinzipielle Frage: Ist dieses durch Prägung erworbene Objekt nun ein Bild - das Bild des Eltern- oder genauer: Folge-Kumpans? Ist das Objekt der Handlung ein Bild? 
Das wäre schon darum ein lohnendes Feld philosophischer Spekulation, weil dieses Objekt als Komplexgestalt oder Bild eben nicht irgendetwas, sondern jemand ist, nämlich „seinesgleichen“, also der Artgenosse. ${ }^{48}$ Ist seinesgleichen ein Bild? Ist er das Bild einer biologischen Art? Wie überindividuell, wie generell ist dieses Bild? Wenn Eltern und Geschwister im Nest das Bild prägen, das dann auf alle anderen Artgenossen übertragen wird, dann müßten, so Lorenz, ,,überindividuelle, artkennzeichnende Merkmale aus dem Bilde der Eltern und Geschwister herausgegriffen und für immer eingeprägt werden“. ${ }^{49}$ Das wird lustig, wo „Um-prägung“, etwa auf das Bild eines Menschen, ins Spiel kommt. Denn diese Umprägung scheint, zur narzißtischen Kränkung des Ornithologen, gar nicht auf einen bestimmten Menschen zu gehen, sondern auf die Art Homo sapiens überhaupt. Lorenz erzählt von einer Dohle, ,,der ein Mensch den Elternkumpan ersetzte und die vollständig ,Menschenvogel' geworden war". Als sie geschlechtsreif wurde, richteten sich ihre „geschlechtlichen Triebe nicht etwa gegen den früheren Elternkumpan, sondern vielmehr mit der vollkommenen Unberechenbarkeit des Sich-Verliebens ganz plötzlich gegen irgend einen verhältnismäßig fremden Menschen, irgend eines Geschlechts, ganz sicher aber gegen einen Menschen. Es scheint sogar, als ob der frühere Elternkumpan als ,Gatte' nicht in Erwägung käme. Woran aber bestimmt so ein Vogel unsere Artgenossen als Menschen?" ${ }^{600}$ Das also geschieht, mit Donna Harraways letztem Buch gesprochen, When species meet. ${ }^{51}$ Lorenz: „Hier harren noch eine ganze Reihe hochinteressanter Fragen der Beantwortung! ${ }^{652}-$

Ding, Bild, Objekt oder Schema: all diese, das Lorenzsche Denken von 1935 tragenden Fragen, werden einen Weltkrieg später aus dem Diskurs der Biologie herausfallen. Nicolaas Tinbergen möchte sie bereits 1948 ein für alle Mal verabschieden, „dieses Wort Schema mit seiner mehr oder weniger mystischen Färbung“. ${ }^{53}$ Tinbergen schlägt vor, nur noch von ,auslösenden Mechanismen“ zu sprechen und das bleibt bis heute der Stand der Verhaltensbiologie..$^{54}$ Lorenz' philosophische Vögel aber fragten nach einer Theorie des biologischen Bildes. Antwort oder Echo werden sie nicht in der Biologie finden. 


\section{Substitution - Übersprung - déplacement. Eine Verschiebung}

Die Reden, Seminare, Schriften Jacques Lacans führen seit den 50er Jahren die Ethologie ins Denken der Psychoanalyse ein. Sie liegt präzise zwischen Lorenz' Bildtheorie und Tinbergens Versuchen. ${ }^{55}$

„Es ist kurios, daß Konrad Lorenz, obwohl er nicht an meinen Seminaren teilgenommen hat, es für angebracht gehalten hat, das sehr hübsche und rätselhafte Bild des Stichlingsmännchens vor dem Spiegel an den Anfang seines Buches zu stellen. “" 1953 war in Paris Konrad Lorenz: Les animaux, ces inconnus erschienen, die Übersetzung von Lorenz' erstaunlichem, direkt nach Heimkehr aus russischer Kriegsgefangenschaft geschriebenem Buch Er redete mit dem Vieh, den Vögeln und den Fischen. Weder auf dem Titelblatt noch im Buch findet sich allerdings das Bild des Stichlings vor dem Spiegel. Die 1952 erschienene deutsche Ausgabe von Nicolaas Tinbergens Instinktlehre $e^{57}$ dagegen trägt als Titelbild Tinbergens Leittier: den Stichling vor dem Spiegel (vgl. Abb. 5). Die Bildunterschrift möchte daraus die halbe Verhaltensbiologie begründen. „Abb. 1 Männchen des dreistachligen Stichlings (Gasterosteus aculeatus) in Drohstellung vor dem Spiegel. Dies angeborene Verhalten (S. 47) hängt von inneren Stimmungsfaktoren (S. 59) und Außenfaktoren (S. 28) ab. Es schreckt andere artgleiche Männchen (S. 166). Stammesgeschichtlich ist es ein ritualisiertes (S. 184) Übersprunggraben (S. 109)." Wo man also mit Lacan lange Zeit nur nach Menschenkind und Affe vor dem Spiegel fragte, da schwimmt des Meisters Denken schon mit dem Stichling, einem Wappentier der frühen Verhaltensbiologie.

\section{Konkordanz Innenwelt - Umwelt}

Im Kontext der Psychose freilich (Seminar von 1955-56) kommt die Frage nach dem Bildverhalten der Tiere nicht aus der Psychologie, und sei es ihrer Krise nach Bühler, sondern aus dem Wahnsinn, la folie, der fünf Jahre vor Foucault explizit das Thema des Seminars ist. Gegen ein „Verstehen“ des Wahnsinns, etwa im Sinn von Karl Jaspers' Psychopathologie ${ }^{58}$, stellt Lacan schlicht die Analyse von „Verhaltenszyklen“, das ist: Ethologie. Der Begriff des Verhaltens tauchte schon 1932 auf, mitten im Horizont des psychiatrischen Denkens der Zwischenkriegszeit. Die Psychiatrie müsse Verhaltenszyklen in der Auseinandersetzung der Psychotikerinnen und Psychotiker mit 
Abb. 5

Männchen des dreistachligen Stichlings (Gasterosteus aculeatus) in Drohstellung vor dem Spiegel. Fontispiz aus Nicolaas Tinbergens Instinktlehre (1952)

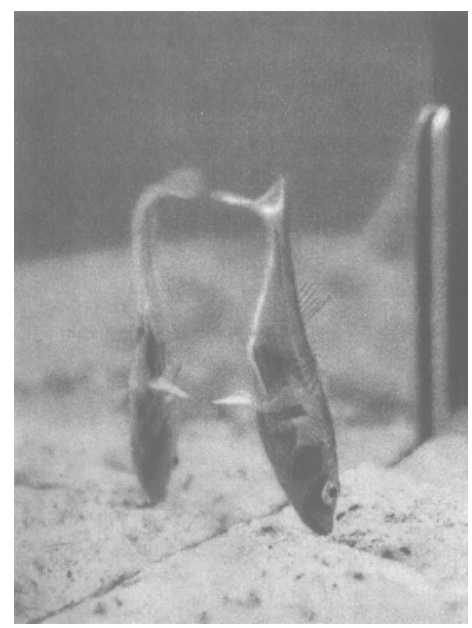

ihrer (sozialen) Umwelt beschreiben, nach Maßgabe von Jakob von Uexkülls Innenwelt und Umwelt der Tiere von 1909/1921. ${ }^{59}$

Der systematische Ausgangspunkt von Lacans biologischem Denken, hier wie anderswo, ist einfach: die „Konnaturalität“ oder „Koaptation“ von - in Lacans Texten immer auf deutsch - Innenwelt und Umwelt bei den Tieren. ${ }^{60}$ Innenwelt und Umwelt sind Teil eines geschlossenen „Funktionskreises“, mit Uexkülls Ausdruck. ${ }^{61}$ Lacan spricht von „instinkthaften Verhaltenszyklen““ ${ }^{62}$ Im Leben der Tiere sei auch die Beziehung zum anderen recht einfach: fressen oder vögeln, quand l'envie les en prend. ${ }^{63}$

Doch taucht auch in den realen und vitalen Befriedigungen beim Tier etwas anderes auf. Man habe die fundamentale Rolle zeigen können, „die das Bild im Verhältnis der Tiere zu ihresgleichen spielt ${ }^{\text {" }}{ }^{64}$ Er listet Tinbergens Standardbeispiele auf: das Profil des Raubvogels, die rote Brust des Rotkehlchens, der rote, rautenförmige Rachen im Fütterungszyklus und schließlich die Auslösung sexueller Verhaltensweisen beim Stichling. ${ }^{65}$ Gerade die Sexualzyklen stünden, so Lacan 1953, „bei den Tieren selbst unter der Abhängigkeit einer gewissen Zahl von Auslösern, Auslösemechanismen, die im wesentlichen der imaginären Ordnung angehören“. ${ }^{66}$ Warum imaginär? Lacan argumentiert, wie so oft, wissensgeschichtlich: weil die Auslöser im Experiment durch künstliche Mittel ersetzbar sind, durch Attrappen, une leurre, einen Köder, eine Falle oder Täuschung. Trotzdem handelt es sich noch immer um die 
Konnaturalität von Innenwelt und Umwelt, sie läuft jetzt nur über auslösende Bilder. Denn daß Auslöser, releasers, déclencheurs überhaupt funktionieren, hat zur Bedingung, daß die Lebewesen an ihre Umwelt ,angepaßt sind“ und mit ihr einen geschlossenen Funktionskreis bilden. ${ }^{67}$

Das Seminar von 1953-54 wird einen speziellen über Bilder laufenden Funktionskreis analysieren: den Tanz der Stichlinge. Le sujet animal, männlich oder weiblich, sei hier ganz gefangen von einer Gestalt (im Französischen auf deutsch): ein duales, in sich abgeschlossenes und reziprokes Verhältnis von Auslösung und Verhalten, Bild und Bild Machen.

In diesem Augenblick findet sich das Subjekt vollkommen mit dem Bild identisch, das die totale Auslösung eines bestimmten motorischen Verhaltens kommandiert, das seinerseits, in einem bestimmten Stil, dasjenige Kommando produziert und dem Partner übermittelt, welches ihn den anderen Teil des Tanzes aufführen läßt. ${ }^{68}$

Doch nur „seinesgleichen“ löse dieses Verhalten aus, das ist: Artgenosse oder -genossin. Die psychoanalytische Mission der Stichlinge geht bei Lacan auf die Freudsche Unterscheidung von Objektlibido und narzißtischer Libido. Bei den Stichlingen falle beides in eins, ja ihr Tanz als solcher sei das Bild dieser conjonction. Lorenz' Frage nach Bild und Objekt, Bild als Objekt und „objektlos ererbtem Trieb“ reformuliert sich:

In der Tat ist die Bindung eines jeden Objekts an das andre [die beiden Stichlinge, $\mathrm{PB}]$ aus der narzißtischen Fixierung an dieses Bild gemacht, denn es ist dieses Bild, und nur dieses, was er [der Stichling, PB] erwartet hat. ${ }^{69}$

Aber das menschliche Subjekt? „Où le situer alors? “70

Lacan situiert es in einer wissensgeschichtlichen Unterbrechung. In einer bekannten, aus der Geschichte der physiologischen Optik verbürgten Anordnung („mein kleiner Apparat“, „den ich erfunden habe"): dem Experiment mit umgedrehter Vase, Blumenstrauß, Hohl- und Planspiegel, endet der Diskurs der Biologie. Die Rede über die Tiere unterbricht sich schließlich selbst, in einem symbolischen Universum von Linien, Buchstaben, Schemata aus dem Physikbuch ${ }^{71}$ und auf der Tafel. Das viermal erneuerte, ergänzte und umgearbeitete Schema vom Experiment mit der umgedrehten Blumenvase wird dem Imaginären der Tiere einen genauen Platz zuweisen: er liege dort, 
wo die Optik und das Auge ein „reelles Bild“ der Vase lokalisieren, also vor dem Hohlspiegel, wo sich dieses reelle Bild in die Welt der realen Objekte einordnet, s'insère. ${ }^{72}$ Beim menschlichen Subjekt dagegen sind Bilder und Objekte sowie alle „Erscheinungen der sexuellen Funktion durch eine eminente Unordnung charakterisiert". Sie läßt sich im Experiment mit der umgedrehten Blumenvase, Version drei, nicht am Ort des reelen Bilds lokalisieren, sondern in einem „Versteckspiel“, jeu de cache-cache, zwischen dem Bild und dem „normalen Objekt“; im imaginären Bild (Planspiegel) des reellen Bilds (Hohlspiegel); in einer halb transparenten Glasscheibe statt des Spiegels, die auch das Objekt durchscheinen läßt - usw. ${ }^{73}$ Mit diesen Bilder-Spielen und ihrer schematischen Verzeichnung beginnt sowohl beim Subjekt als auch in der Anordnung der Diskurse die Ordnung des Symbolischen.

Zwei Jahre später wird dem Bruch von der Biologie des Imaginären zum symbolischen Universum ein Zwischenspiel in der Biologie selbst vorgelagert sein. Das ist: eine spezielle Situation tierischen Verhaltens, auf die Lacan immer wieder zurückkommt. Mitten im biologischen Bild als Medium konnnaturaler Funktionskreise ereigne sich hier plötzlich etwas anderes: eine Verschiebung, un déplacement, a displacement.

\section{Displacement}

„Verschiebung“ ist an dieser Stelle kein Lacanismus, aufgespannt zwischen Freuds Verschiebungsarbeit und Roman Jakobsons shifters. Es ist ein Stück Wissenschaftsgeschichte. Displacement reaction bezeichnet im Jargon der Ethologie einen bestimmten Moment im Verhalten mancher Tiere.

Die Drohgebärde des Stichlings nämlich ist, näher betrachtet, merkwürdig. Der Fisch steht senkrecht und, so Tinbergen 1937, „der nach unten gerichtete Kopf stößt ruckweise in den Boden“74 (vgl. Abb 6 und 3). Diese Bewegung ist die gleiche wie die bei der Suche nach Nahrung im Sand. Sie ist auch die gleiche wie die beim Graben, wenn das Männchen im Sand die Mulde für das Nest herstellt. 1940 nun machen unabhängig voneinander die zwei Holländer Adriaan Kortlandt und Nicolaas Tinbergen eine Reihe merkwürdiger Beobachtungen. ${ }^{75}$ Sie sehen zwei Hähne kämpfen und auf dem Höhepunkt des Kampfes fangen die Hähne plötzlich an, im Sand nach Körnern zu picken; sie sehen zwei Vögel, zwei Säbelschnäbler, kämpfen und plötzlich steckt der eine Vogel seinen Kopf ins Gefieder als würde er schlafen; sie sehen zwei Entenerpel kämpfen und plötzlich, mitten im Kampf, fängt der eine an, sich 


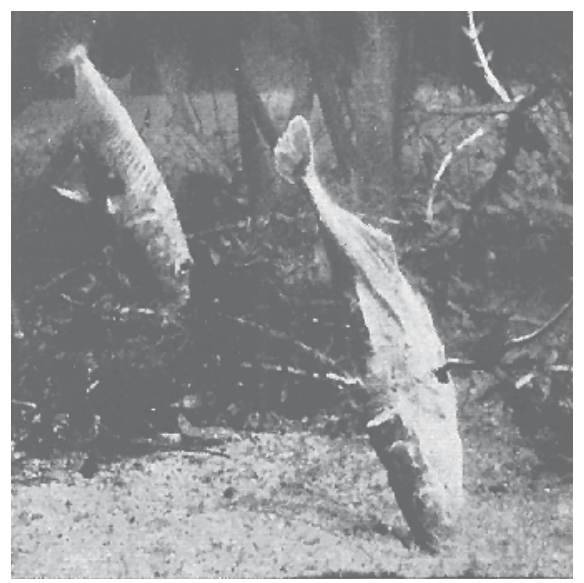

Abb. 6

\section{Das drohende Männchen} fixiert das Modell. aus Nicolaas Tinbergens "Die Übersprungsbewegung", Zeitschrift für Tierpsychologie (1940)

demonstrativ die Federn zu putzen und zu glätten. Kortlandt und Tinbergen stellen zunächst die Hypothese auf, hier würden zwei widerstrebende Triebe, Flucht und Angriff, aufeinander treffen $^{76}$ und entwerfen drei Auswege aus dem Dilemma: 1. eine kompromissuelle Handlung; 2. eine wechselnde Folge von Fliehen und Angreifen; oder 3. der Ausweg, plötzlich etwas ganz anderes zu tun. Man spricht zunächst von irrelevant movements oder sparking over actions, Übersprungsfunken von einer Handlungsbahn in die andere, oder auch von substitute activity. Tinbergen, Huxley, Thorpe werden das Phänomen schließlich auf den Namen displacement acitivity taufen. ${ }^{77}$ Tinbergen konstruiert mit seinen Stichlingen 1940 und 1946 dazu zwei Experimente.

Wenn ein Stichlingsmännchen in der Brunftzeit Revierverhalten entwickelt, verjagt es fremde Stichlinge aus dem eigenen Territorium; im fremden Territorium dagegen flieht es sofort, wenn angegriffen. Probleme entstehen nur an der Grenze zweier Territorien. Oft sieht man hier zwei Nachbarn endlos und hektisch hin- und herschwimmen. Oder in manchen Fällen dann auch drohen. Damit setzt Tinbergens experimentelle Frage ein: Kann man „ein Tier, das in Angriffsstimmung ist, durch [künstliche, PB] Erregung des Fluchttriebes zum Drohen bringen“", das ist: in die senkrechte Lage? ${ }^{78}$ Tinbergen stellt also eine Stichlingsattrappe im vollen Prachtkleid im Revier des Stichlings auf (vgl. Abb. 6). „Läßt man nun die Attrappe zurückkämpfen, indem man das Männchen fortwährend mit ihr anstößt, so hat das anfänglich nur stärkere Reizung des Kampftriebes zur Folge, bald aber Reizung des 
Fluchttriebes. Das Männchen benimmt sich dann, als wäre es von einem Eindringling besiegt worden und verkriecht sich irgendwo zwischen den Pflanzen. Hält man die Attrappe nun ruhig, so kommt das Männchen allmählich wieder zum Vorschein und greift auf einmal wieder an. Jetzt aber wechselt es mit Kämpfen und Drohen. ${ }^{\text {"79 }}$ Drohen läßt sich also, so die erste Erkenntnis, durch eine Attrappe provozieren.

1946 treibt Tinbergen die Sache ins Extrem. Er sperrt fünf Stichlinge auf engem Raum zusammen. Sofort setzt ein wildes Drohen und Fliehen und Kämpfen und wieder Drohen ein. Diese Aktivität hinterläßt Spuren: das Bild eines Felds mit Löchern oder Trichtern (vgl. Abb. 7). Denn bei jedem Drohen gräbt der Stichling ein Loch im Sand als würde er für das Weibchen ein Nest vorbereiten. Es sind richtige, tiefe Löcher und die Handlung keine schwächere Form der originalen Grabhandlung, ja sie ist sogar verstärkt (einer der Stichlinge etwa gräbt eine riesige Grube). Die Löcher markieren nun das Territorium des Stichlings wie Trichterfelder die Frontlinien des Ersten Weltkriegs. Bildunterschrift: „Gestrichelt: Grenzen, denen entlang die Revierbesitzer einander androhen; dazwischen Niemandsland.“

Abb. 7
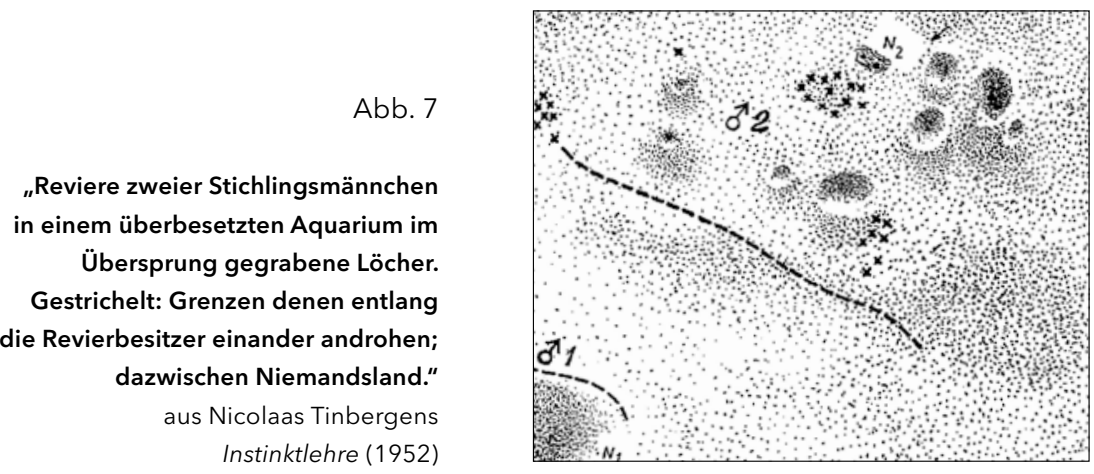

\section{Lacans Stichling}

Wie verschoben kommt diese Verschiebung als Bildverhalten des Stichlings in Lacans Theorie des Imaginären an?

Zunächst wird sie als Verschiebung zwischen zwei verschiedenen Verhaltenszyklen gedeutet: aus dem „Kampfzyklus“ in den „Zyklus des Sexual- 
verhaltens". Wenn bei den Vögeln, so Lacan 1953, mitten im Kampfzyklus „plötzlich einer der Kämpfenden beginnt, sich die Federn zu glätten“, dann scheint hier „das jähe Auftauchen eines Segments aus dem Paradeverhalten zu beobachten“. ${ }^{80}$ Denn jedes Paradeverhalten, so wäre die biologische Behauptung, ist ein sexuelles (und es gäbe keine genuin kriegerischen Paraden). Im Seminar über die Psychosen drei Jahre später beschreibt Lacan dann ein weiteres Mal und ohne Nennung von Namen und Quellen Tinbergens Versuche: das Revier des Stichlings und den Tanz, das röhrenförmige Nest, wie „ein kleiner Tunnel“ ${ }^{81}$ „Aber es gibt da noch etwas, das nicht leicht zu erklären ist, daß nämlich das Männchen, wenn alles vollbracht ist, noch Zeit findet, hier und da eine Menge kleiner Löcher zu machen. “82

Die Löcher des Stichlings - bei deren falscher Lokalisierung im Verhaltenszyklus wir uns nicht aufhalten ${ }^{83}$ - ereilt dann eine Verschiebung des Diskurses: in Philosophiegeschichte. ${ }^{84}$ Am Ende von Das Sein und das Nichts entwickelt Jean-Paul Sartre eine Reihe von fundamentalen, qualitativen, an Dinge gebundenen Erfahrungen der Seinserschließung: das Klebrige, das Dunstige, das Teigige und am Ende das Loch, die Löcher, „Löcher im Sand und in der Erde“.$^{85}$ Vor aller psychoanalytischen Deutung, so Sartre, gebe es das Loch als fundamentales Phänomen. Gerade im Leben der Kinder sei es unübersehbar präsent. „Alle Arten von Löchern (Löcher im Sand, in der Erde, Grotten, Höhlen, Vertiefungen)" übten eine Anziehung auf Kinder aus. Die Löcher sind entweder das, was zu stopfen ist, bis hin zum Daumen Lutschen, ja zum Essen als Stopfen eines Lochs. Oder die Löcher gehören dem „umgekehrten Trieb an, dem Trieb, Löcher zu bohren“ ${ }^{86}$ Die ganze Sexualisierung des Lochs, die die Psychoanalyse versucht, setze, so Sartre, die qualititative Urerfahrung des Lochs voraus. ${ }^{87}$ Lacans Echo: „diese Scheinmanifestationen der Negativität [...], nun, ich glaube, daß diesbezüglich das Stichlingsmännchen nicht im Rückstand ist. " ${ }^{\text {"88 }}$

Die Sartre-Verschiebung führt bei Lacan, Sartres Gedanken entwendend, ins Drohverhalten des Stichlings ein Sexualverhalten ein, was Kortlandt und Tinbergen nicht tun. Das Löcher Graben des Stichlings in der Drohgebärde wird als Teil eines Sexualzyklus verstanden, als „Grenzreaktion zwischen Eros und Aggressivität, die durch das Bohren der Löcher angezeigt wird“" 89 Biologisch müßte man also das Graben und Nestbauen, das vor dem Hochzeitstanz stattfindet, bereits als Teil des Zyklus der Verführung begreifen. Das bleibt prekär. Zumal es andere, vielleicht schlagendere Beispiele dafür gibt als den Stichling, wenn etwa Kortlandt von Vögeln berichtet, die sich mitten im Kampf auf ihren Gegner setzen und Kopulationsbewegungen ausführen. ${ }^{90}$ 
Wie dem auch sei: Weil nur in den sexuellen Registern „die Ordnung der imaginären Befriedigung", de la satisfaction imaginaire, $\mathrm{zu}$ finden ist ${ }^{91}$, darum findet gerade hier die Verschiebung heraus aus der vitalen Notwendigkeit, dem Kampf der Arten auf Leben und Tod, in das Register des Bild Machens statt. Das sexuelle Verhalten biete auch bei den Tieren die größte possibilité de déplacement, also Freiheit, überhaupt ein Bild darzubieten und zugleich Freiheit, „ein falsches Bild“ darzubieten. ${ }^{92}$ „So gehen wir davon aus, daß ein Verhalten imaginär werden kann, wenn seine über Bilder erfolgende Weichenstellung und sein eigener Wert als Bild für ein anderes Subjekt es zu einer Verschiebung aus dem Zyklus heraus befähigen, der die Befriedigung eines natürlichen Bedürfnisses sichert. “93

Mit dieser Verschiebung aber beginnt, so Lacan, eine andere Ordnung. Das „verschobene instinktive Verhalten beim Tier“ gebe „den Aufriß“, die erste Andeutung, l'ébauche, „eines symbolischen Verhaltens“.${ }^{94}$ Rückübersetzt in die Wissensgeschichte der Biologie: das Graben, im Zyklus der Reproduktion eine funktionale Triebhandlung, nimmt einen imaginären Wert für ein anderes tierisches Subjekt an und eben darin zeigt sich die erste Andeutung einer Symbolbildung. Im Unterschied also zur Ethologie nach Lorenz und Wickler, nach der sich Symbolhandlungen und Signale durch mimische Übertreibung einer einzelnen Intentionshandlung ins Unwahrscheinliche entwickeln, ereignet sich hier das Symbolische in der Verschiebung eines ganzen Handlungszyklus ins Bildhafte: in Parade, Demonstration, Verführung. ${ }^{95}$

\section{Ein sekundärer Nesthocker}

Das alles findet schon beim „tierischen Subjekt“ statt. ${ }^{96}$ Über den Bruch zum menschlichen Subjekt ist damit noch wenig gesagt. Dieser Bruch gründet bei Lacan selbst auf einem Bild oder, genauer, der Reduktion auf ein einziges Bild: das Spiegelbild des eigenen Körpers. Dieses Bild gehört nicht nur der Geschichte einer alten Kulturtechnik an, die eines geschichtlichen Tages ebenso in der physikalischen Optik wie in bürgerlichen Schlaf- und Kinderzimmern ankommt. Auch das Bild im Spiegel hat eine Biologie.

Tinbergen stellt irgendwann in den 40er Jahren ein Stichlingsmännchen vor den Spiegel (vgl. Abb. 5). ${ }^{97}$ Lacan: „Was macht es?“ Es macht das gleiche wie vor der Attrappe von seinesgleichen gleichen Geschlechts: stellt sich senkrecht und tut so als würde es Löcher bohren. „Anders ausgedrückt, sein 
Bild im Spiegel läßt es nicht gleichgültig ${ }^{698}$, auch wenn es nicht wirklich in den erotischen Verhaltenszyklus eintritt, also tatsächlich Löcher bohrt. Das Spiegelbild als Köder versetzt den Stichling in eine angetäuschte, verwirrte Lage, ein déreglement, das nicht mehr in der Koaptation von Innenwelt und Umwelt aufgeht.

Beim Menschenkind aber greift das Spiegelbild als Köder in eine Lage ein, die selbst schon auf eine fundamentale Weise deplaziert ist. Der Diskurs über sie kommt aus der Biologie, das ist: der vergleichenden Entwicklungsgeschichte der Säugetiere. ${ }^{99}$

Biologisch nämlich ist (einer frühen Arbeit Adolf Portmanns folgend) der Mensch schon darum kein „echtes Säugetier“100, weil er weder zur Gruppe der Nesthocker noch zur Gruppe der Nestflüchter gehört. Eichhörnchen, Iltis, Marder gebären nackte, hilflose Junge, deren Augen sich erst Wochen nach der Geburt öffnen, als feuchte Organe also vor Austrocknung an der Luft geschützt sind. Elefanten, Kälber, Giraffen dagegen können schon in der ersten Stunde nach ihrer Geburt stehen und der Herde folgen oder wie die Schimpansen sich aktiv ins Fell der Mutter klammern. Ein Dogma der vergleichenden Entwicklungsbiologie besagt, daß die Nestflüchter nach Aktionsradius, Bauplan, Gehirngröße die evolutionär höher organisierten Formen seien. Ein Beweis: Nestflüchter machen im Mutterleib die Phase der verschlossenen Sinnesorgane durch, die beim Embryo, der im Amnion ohnehin gegen Austrocknen geschützt ist, rein funktionell schwer zu erklären ist. ${ }^{101}$

Und das neugeborene Menschenkind? Nackt und schutzlos scheint es ganz und gar auf Hilfe aus der Umgebung angewiesen, wie ein typischer Nesthocker. Aber der erste Eindruck täuscht. Denn näher besehen trägt das Kleine alle biologischen Kennzeichen eines Nestflüchters: Augen und Nase stehen weit offen, das zentrale Nervensystem gleicht dem eines Fohlens, die motorischen Fasern im Rückenmark entsprechen schon denen des Erwachsenen und auch die unkoordinierten Bewegungen sind nicht nur Hilflosigkeit, sondern aktives und „freies Spiel der Gliedmaßen“, also „Spielraum“. ${ }^{102}$ Nur ist dieser Nestflücher, das Menschenkind, genau ein Jahr zu früh geboren, um ein „echtes Säugetier von menschenartiger Organisation" ${ }^{103}$ zu sein. Darum ist es so schutzlos. Die Biologie spricht von einem „sekundären Nesthocker“, dessen erstes Lebensjahr ein „extra-uterines Frühjahr“ sei. ${ }^{104}$ Durch dieses erste Jahr unterscheide sich die Ontogenese des Menschen strukturell von der aller Säugetiere. Die Einebnung seiner Besonderheit im Dispositiv von Abstammungen, Stufenmodellen, biogenetischem Grundgesetz zeige nur, so Adolf 
Portmann, das „Unverbindliche der Abstammungsdiskussionen““. ${ }^{105}$ Dagegen setzt er, gut lamarckistisch und phänomenologisch, die „Einheit eines Entwicklungsgeschehens" ${ }^{\text {"106: }}$ die strukturellen Gleichzeitigkeiten, von stabiler Lage, also Stehen, und Nachsprechen etwa; die aktiven Instabilitäten, das drängende Herstellen immer neuer Situationen, Herumprobieren, Lallen, schließlich Nachahmen ${ }^{107}$; die daraus langsam entstehende ,intensive Kenntnis des eigenen Körpers, das Verfügen über die Bewegungen der Arme und Beine [...], aber auch die Macht über die Bewegungen der Lautorgane“108, usw.. Das Menschenkind wachse im ersten Lebensjahr eben nicht abgeschlossen im Mutterleib und durch „Selbstdifferenzierung“ heran, sondern „durch Kontakt mit der Umgebung“". Und das heißt, nach Portmanns folgenreicher Bemerkung: durch „ungezählte „Ereignisse“, die einmalig sind“. In diesen einmaligen, biologisch nicht determinierten, artmäßig nicht gleichförmigen Ereignissen gründe am Ende das „Geschichtliche“ als solches, also auch eine Geschichte des Subjekts. ${ }^{109}$

Am 3. August 1936 interveniert ein junger Psychiater aus Paris auf dem der Kinderanalyse gewidmeten Kongreß der Internationalen Psychoanalytischen Vereinigung in Marienbad in diesen Diskurs über das erste Lebensjahr. Weniger durch Herumprobieren, wie die Biologen es wollen, gewinne das Menschenkind eine erste Berrschung, maîtrise, des eigenen Körpers ${ }^{110}$ als durch Angleichung an Bild und Gestalt von seinesgleichen. Darin entstehe etwa ab dem sechsten Monat, der Mitte des extra-uterinen Frühjahrs, bis zum etwa achtzehnten jene Form im emphatischen Sinn, über die erst die eigenen Bewegungen bis in ihre Statik hinein koordinierbar werden. ${ }^{111}$ Die Intervention hat ihr Besonderes auch darin, daß sie anders als Psychiater, Psychologen, Psychoanalytiker sonst ${ }^{112}$ das Bild vom eigenen Körper als einen biologischen Diskurs einführt. Nicht nur weil das Bild in eine biologisch definierte déhiscence oder béance fällt, eben die „,normalisierte Frühgeburt“, in der Innenwelt und Umwelt auseinanderklaffen, oder weil, von der Ethologie her gesehen, jedes biologische Bild eine Attrappe, ein Köder, une leurre ist. Sondern darum, weil erstens die Angleichung ans Bild von seinesgleichen biologisch verbürgt scheint: in der Gonadenreifung der Tauben beim Anblick ihres Artgenossen, der auch ein Bild sein kann, oder im Übergang der Wanderheuschrecken vom solitären Stadium zum Schwarmstadium, ausgelöst durch den Anblick eines Artgenossen mit artspezifischen Bewegungen. ${ }^{113}$ Und weil sich zweitens der Mechanismus selbst der Angleichung ans ganze Bild vom eigenen Körper auf ein biologisches Phänomen berufen kann. Lacan setzt hier gegen den 
Nachahmungstrieb beim Affen die Mimikry der Insekten, wie sie der Philosoph Roger Caillois seit den surrealistischen 30er Jahren beschreibt. Deren lamarckistische Quintessenz besagt, daß in der heteromorphen Angleichung einer Falterart an eine andere, einer Heuschrecke an ein grünes oder auch, in einer äußersten Übertreibung oder Hypertelie, an ein trockenes, verwelktes, mit Schimmel besetztes und angefressenes Blatt nicht die Selektion durch Freßfeinde wirkt, sondern eine pathologische Verrückung in der Natur selbst. Wie in manchen psychasthenischen Zuständen gewinne hier der Raum, normalerweise Garant der Trennung von Organismus und Umgebung, eine solche Dichte und Macht über die Lebewesen, daß sie in ihm aufgehen, ihm gleich werden. Eben diesen, bei Caillois in allen biologischen Einzelheiten ausbuchstabierten Prozeß ruft Lacan auf, um die Macht des einen Bildes zu begründen ${ }^{114}$, über das im Unterschied zur reichen Bilderwelt der Tiere das Imaginäre des Menschen laufe.

Die Beziehung zum eigenen Körper charakterisiert beim Menschen das letztlich reduzierte, [das ist: im Vergleich zu den Tieren reduzierte, PB] aber irreduzible Feld des Imaginären. Wenn irgendwas beim Menschen der imaginären Funktion entspricht, wie sie beim Tier wirksam ist, so ist es all das, was ihn in elektiver, aber stets äußerst schwer erfaßbarer Weise zur allgemeinen Form seines Körpers in Beziehung setzt, wo dieser oder jener Punkt - beim Tier als releaser wirksam - als erogene Zone bezeichnet wird. ${ }^{115}$

Auch wenn Serge Leclaire die Artikulation dieser Zonen dereinst direkt als „buchstäbliche Ordnung“" ansprechen wird: in Lacans Theorie des menschlichen Subjekts ist die Beziehung zur Bild-Attrappe des eigenen Körpers, jenem Doppelgänger oder ,andern“ mit kleinem a, zugleich die Eröffnung der Sprache und sie liegt vor der Sprache. „Am Ursprung, vor der Sprache, à l'origine, avant le langage, existiert das Begehren nur auf der einzigen Ebene der imaginären Beziehung des Spiegelstadiums, projiziert, entfremdet im andern. " ${ }^{116} \mathrm{Im}$ ganzen Unterschied zum Tier nämlich etabliert die imaginäre Beziehung beim Menschen nicht einen geschlossenen Funktionskreis im Dispositiv der Adaptation, sondern eine „Spannung“, das ist: eine imaginäre Konkurrenz auf Leben und Tod zwischen dem Ich und seinem andern als Bild. „Sie hat keinen anderen Ausweg - wie Hegel uns lehrt - als die Zerstörung des andern. “" ${ }^{117}$

Struktur und Lösung dieser zerstörerischen Bildfaszination des menschlichen Subjekts demonstriert das Seminar über die Psychosen an einem Gedan- 
kenexperiment. In dessen Mitte taucht ein sehr merkwürdiges Phylum von Tieren auf. Man nehme an, was niemals der Fall sein wird: die Beziehung des Menschen zu seinesgleichen beschränke sich auf die imaginäre Bildrelation, diese ,assimilierende und dissimilierende Fesselung“" capture. ${ }^{118}$ Man entwickle dann aus dem gedachten Experiment ein durchführbares. In den 50er Jahren lernt Lacan die kleinen, biologisch streng nach Linné, also binär klassifizierten Wesen der Gattung Machina des amerikanischen Kybernetikers William Grey Walter kennen: M. sopora, M. labyrinthea, M. speculatrix. ${ }^{119}$ Die kleinen Maschinen ähneln zwar Stichlingen kaum und Schildkröten nur entfernt, doch lassen sich auch mit ihrer Hilfe, so Lacan im direkten Anschluß an den Stichling, bestimmte Verhaltenszyklen experimentell untersuchen. Machina speculatrix etwa, ausgestattet mit Lichtsensoren und Leuchtorganen, steht schon bei Grey Walter vor dem Spiegel: sie schaltet nach Maßgabe ihres im Spiegel gesehenen Lichts ihr Licht aus, gerät also in eine klassische singing condition - „das Geschöpf zögert vor einem Spiegel und flattert und

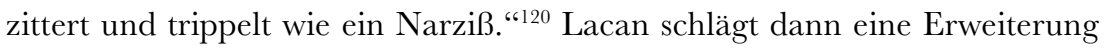
der Maschine vor. Man konstruiere einen Vetreter der Gattung, der die rechte Pfote nur dann synchronisiert mit der linken heben kann, wenn „ein photoelektrischer Empfangsapparat das Bild einer anderen Maschine überträgt, die im Begriff ist, harmonisch zu funktionieren“, sprich: das gleiche zu tun. ${ }^{121}$ Man nehme schließlich einen ganzen Schwarm solcher Maschinen und werfe sie auf die freie Fläche einer Autoscooterbahn. Da jede Maschine durch den Anblick der anderen reguliert wird, ,,ist es mathematisch nicht unmöglich“, daß irgendwann alle Maschinen aufeinander zustürzen und sich „im Zentrum konzentrieren“, verkeilen, gegenseitig blockieren: im Bild einer allgemeinen Karambolage, une collision, un écrabouillement général.

„Aber, Dieu merci, das Subjekt ist in der Welt des Symbols, das heißt in einer Welt von anderen, die sprechen, “122 und nicht in einer Welt „blockierter Maschinen“, wie Lacans Kybernetismus geruht, die Tiere auch anzusprechen. ${ }^{123}$ Dem merkwürdigen Subjekt aber, le sujet bicornu, das der Mensch ist, wäre nicht einmal ein „animales Leben“ möglich ohne die „überlagerte“ symbolische Ordnung. ${ }^{124}$ Wenn also dieses Subjekt in „der Erfahrung“", das ist: Übertragung, durch seine Verwechslungskomödien, täuschenden Reden, scheinbaren oder echten Kontrakte in die Kommunikation eintritt und damit in die Anerkennung des anderen als System der Sprache, so tritt das Subjekt als Wissensgeschichte aus der imaginären Ordnung der Tiere, also dem Wisssen der Biologie, in die Geschichte buchstäblich schematisierter Anordnungen 
von physikalischer Optik oder Maschinen, jener „radikalsten symbolischen Aktivität beim Menschen“ ${ }^{125}$ Einer biologischen Bildwissenschaft wüchse damit ein wissenstheoretischer Standort zu, den sich die Biologie selbst wohl kaum träumen ließe. 


\section{Anmerkungen}

1 Vgl. Foucault 1961/1973: 531, 535.

2 Vgl. Kadi 1999. Vgl. auch Blümle/von der Heiden 2005.

3 Wir kennen diese Romane bis heute nicht vollständig, weil Lacan sie Marguerite Pantin, sehr unfein, einfach abgenommen hat.

$4 \quad$ Vgl. Dalí 1933/1974: 200 f.

5 Lacan 1932/2002: 320.

6 Lacan 1938/1980: 59.

7 Vgl. Lacan 1936-1949/1973.

8 Lacans frühe Schrift „Jenseits des Realitätsprinzips“ von 1936, in der sich eine längere Abhandlung über die „Probleme des Bildes“ findet (Lacan 1936/1994: 17 -22), endet mit der Ankündigung einer Forstsetzung ,über die Realtität des Bildes und die Formen der Erkenntnis“ (ebd.: 37). Sie wird nie geschrieben. Rätselhafte Begründung von 1966: „Deshalb haben wir unserm Aufsatz über das ,Realitätsprinzip‘ die Fortsetzung, die er ankündigte, nicht gegeben, weil er sich an den Gestaltismus [i. Org. dt.] und die Phänomenologie halten muß.“ (Lacan 1966/1980: 12).

9 Vgl. Freud 1895/1950: vor allem 325-327 und 334-336.

10 Lacan 1953/2002: 21.

11 Siehe den Beitrag von Markus Klammer in diesem Band, 241-256.

12 Lacan 1955-56/1981 (frz.): 17. - Lacan entwendet einen Buchtitel Henri Piérons, von 1929 bis 1951 Professor für Physiologie de la sensation am Collège de France: La sensation, guide de vie, Paris 1945.

13 „Mimikry“ wäre dann, in einer Erweiterung des Begriffs, das Leben der Tiere in und mit dem Bild Machen, Bild Geben, Bild Empfangen überhaupt.

14 So visionierte Bernhard Siegert (Weimar) einmal.

15 Über den Stichling heißt es dort: „Und da gerade die Männchen es sind, die vor Verliebtheit nicht aus noch ein wissen, deutet schon ihr farbenblühendes Kleid es der Erwählten an: ,Ich liebe dich, ich brenne nach dir, ich sterbe vor Begier, dich zu besitzen!““ Der, so Francé-Harrar, „Beweis“ dafür: wird der Stichling abgewiesen oder unterliegt im Kampf, verliert er sofort seinen feuerfarbenen Bauch und wird wieder „grünlich trüb“ (Francé-Harrar 1926: 82).

16 Hegel 1830/1970: 433.

17 Vgl. etwa Lorenz 1935: 186; oder Lorenz 1949/1970: 91.

18 Vgl. Heidegger 1935/1987.

19 Bei Uexküll sind die Schemata meist physiologisch-technische Schaltpläne von 
Nerven und heißen auch „Gegenwelt“ oder „Innenwelt“. Die Gegenwelt also besteht aus Schemata (vgl. etwa Uexküll 1909/1921: 168).

20 Claude Elwood Shannons „Mathematische Theorie der Kommunikation“ (1948) behandelt bekanntlich Zeichen, Worte, Bilder, Töne, wenn und insofern sie übertragen, also gesendet werden, ganz ohne Semantik als bloße Frage von „Erwartungswahrscheinlichkeiten" und folgt darin einem mathematischen Ansatz des russischen Wahrscheinlichkeitstheoretikers Andrej Andrejewitsch Markov von 1913.

21 Vgl. Hofer 2002.

22 Bühler 1929: 51.

23 Darwins Theorie, daß die Ausrucksbewegungen frühere zweckgerichtete Bewegungen seien (beissen - Zähne fletschen) wird damit ebenfalls hinfällig (Bühler 1929: 33 f.).

24 Bühler 1929: 38.

25 „Nun wissen wir gar nichts von einem Innern, einem Zumutesein materieller Systeme. Aber man gebe uns eines von der Art, wie sie die Menschen für die Zwecke ihrer Mitteilungen in die Ferne konstruiert haben, im Betrieb zur Untersuchung, und wir wollen an ihm rein mit den Mitteln des Technikers über die Art der Koppelung und die Angelegenheit der Steuerung allerhand wichtige Erkenntnis gewinnen." (Bühler 1929: 43).

26 Der Begriff der Auslösung stammt aus der letzten, kleinen Schrift des Arztes und Erfinders des Ersten thermodynamischen Hauptsatzes, Julius Robert Mayer, „Über Auslösung“ (1876). Mayer beschreibt unter diesem Titel Prozesse, in denen kleinste Kräfte größte Wirkungen hervorbringen (Funke - Explosion, Abzug - Schuß, usw.) und die darum der Äquivalenz der Energieformen zu widersprechen scheinen. Der Begriff nimmt von dort eine wechselvolle Geschichte über Nietzsche, Wilhelm Ostwalds und Alwin Mittaschs „Energetik“ bis in heutige, medienhistorische Versuche, eine Urgeschichte der Kybernetik zu entwerfen.

27 Bühler 1929: 43.

28 Vgl. Bühler 1929: 40.

29 Bühler 1929: 39.

30 Bühler 1929: 47.

31 Hofer macht vor allem darauf aufmerksam, daß Bühlers „probabilistisches Konzept des ,Spielraums“" seit 1918 diesen Filter im Dispositiv der Darwinschen „Selektion“ begreift: Selektion einiger Handlungen aus einem „Überfluß“" spielerischer Versuche (Hofer 2002: 138-140).

32 Donald Campbell etwa versucht dies in den 80er Jahren. Jede Anpassungshand- 
lung des multidimensionalen Homöostaten namens Tier braucht dann Referenzsignale und Schablonen für den Zielzustand. ,Wo es sich um gegenseitige Beeinflussung von anderen Tieren oder Gegenständen handelt, nehmen diese Kriterien den Charakter von ,Bildern` an, und eine phänomenologische Dimension wird hinzugefügt. Hierher gehört Lorenz’ Begriff des ,Auslösers““ (Donald Campbell, „Neueinführung von Konrad Lorenz in die Psychologie“, in: Konrad Lorenz, Gespräche mit Richard I. Evans (und anderes), hg. Richard I. Evans, Berlin 1977, S. 75-104, 80, zit. bei Hofer 2002: 153).

33 Lacan 1953-54/1975 (frz.): 254 und 255. - Das Recht, so Bühler, bestrafe den Meineid eines Zeugen auch dann, wenn er schließlich genau das Richtige trifft; es läßt den Eid in bestem Wissen straffrei, auch wenn er schließlich das Falsche sagt. Das Recht kommt Bühler schon darum in den Blick, weil er nicht vom wissenschaftlichen Satz, sondern vom ,lebendigen Sprachverkehr“ ausgeht (Bühler 1929: 48). Man müßte nur „Überzeugung des Sprechers“ und „Sachverhalt“ nicht mehr wie Bühler in zwei „Sinndimensionen“ trennen und das Rechtssystem selbst zum dritten Term machen, um bei einem „Quellpunkt“ lacanschen Sprachdenkens zu landen.

34 Diese Frage hat in den 60er Jahren vor allem der Nachfolger von Konrad Lorenz im Max-Planck-Institut für Verhaltensphysiologie in Seewiesen, Wolfgang Wickler, weitergeführt, etwa in seinen Forschungen über die Signalbildung an den Eiattrappen von Buntbarschen der Gattung Haplochromis (vgl. Peter Berz, „Die Kommunikation der Täuschung. Eine Medientheorie der Mimikry“, in: Mimikry. Gefährlicher Luxus zwischen Natur und Kultur, hg. Andreas Becker, u. a., Schliengen 2008, S. $27-44)$.

35 Lorenz 1950/1965: 448.

36 Lorenz 1950/1965: 446.

37 Lorenz 1935: 143.

38 Lorenz 1935: 140.

39 Lorenz 1935: 140 f..

40 Vgl. Lorenz 1935: 141, u.a.

41 Zitat ohne Angabe bei Lorenz 1935: 166.

42 Lorenz 1935: 164.

43 Lorenz 1935: 174. - Ausnähbilder bestehen aus vorgestanzten Löchern, die (wie beim Malen nach Zahlen) durch Nadel und Faden verbunden werden.

44 Spektakulärstes Beispiel: Als bei einer Überschwemmung des an der Newa gelegenen Instituts Iwan Pavlovs alle seine Hunde in der eiskalten Newa landeten, vergaßen sie sofort sämtliche Konditionierungen. 
45 Lorenz 1935: 167 f.. - Hans Spemann, den sein Freiburger Kollege Martin Heidegger 1929/30 zum Urtyp des „führenden Forschers“ ernannte, erhält genau im Jahre 1935 für die Entdeckung des „Organisatoreffekts“ den Nobelpreis für Medizin.

46 Vgl. Portmann 1948/1965: 118-122.

47 Lorenz 1935: 169, u. a.

48 Zum Sprachgebrauch von „seinesgleichen“ oder „,ihresgleichen“ vgl. Lorenz 1936: 163, 167.

49 Lorenz 1935: 172.

50 Lorenz 1935, Hervoherbungen von Lorenz.

51 Vgl. Haraway 2008.

52 Lorenz 1935: 172.

53 Tinbergen 1948: 31.

54 Vgl. etwa McFarland 1985/1989: 332 ff., Kapitel 20.2: „Der angeborene Auslösemechanismus".

551953 in einer Rede über das Symbolische Imaginäre Reale, 1954 in den Sitzungen über die Topik des Imaginären des ersten Seminars und 1956 im Seminar über Psychose und Wahnsinn (Lacan 1953/2006, 1953-54/1990 und 1955-56/1997).

56 Lacan 1955-56/1997: 114.

57 Englisch: The study of instinct von 1950. Es handelt sich um Tinbergens Vorlesungen an der Columbia-University im Jahre 1947.

58 Mit der Lacan 1932 noch arbeitet und die 1956 nur noch mit pädagogischem Spott überzogen wird.

59 Lacan 1932/2002: 334 (und Anmerkung 21); vgl. auch 50 (und Anmerkung 28).

60 Lacan 1938/1980: 59. Vgl. auch den Ausdruck „,coaptation imaginaire“ in: Lacan 1954-54/1975 (frz.): 190. Co-aptation (statt ad-aptation) ist ein Wort aus der Chirurgie und bezeichnet die Annäherung und Verheilung der zwei Ränder einer Wunde oder eines gebrochenen Knochens.

61 Vgl. etwa Uexküll 1909/1921: 44-49. - Lorenz wird den Ausdruck übernehmen (vgl. etwa Lorenz 1935: 142, u. a.).

62 Lacan 1955-56/1997: 112. - Im Zweiten Seminar Lacans wird daraus, die Tiere ganz ins technische Dispositiv des Seminars einschreibend, ein „Reflexbogen“.

63 Lacan 1955-56/1981 (frz.): 108.

64 Lacan 1955-56/1997: 112.

65 Vgl. etwa Tinbergen 1948.

66 Lacan 1953/2006: 21.

67 Der eine Kreis kann, folgt man Lorenz, auch aus getrennten Funktionskreisen be- 
stehen. Wie mehrere Auslöser nur zufällig in einem Ding, so sind Füttern, Folgen auf dem Boden, Folgen in der Luft nur zufällig in einen einzigen Artgenossen vereint. Sie könnten auch auf verschiedene Artgenossen, ja sogar auf Tiere anderer Arten verteilt sein (vgl. etwa Lorenz 1936: 144 f.).

68 Lacan 1953-54/1990: 177.

69 Lacan 1953-54/1990. - Oder schlichter formuliert: „Das Tier läßt ein reales Objekt mit dem Bild, das in ihm ist, koinzidieren." (ebd.: 178)

70 Lacan 1953-54/1975 (frz.): 158

71 In diesem Fall Henri Bouasse, Optique et photométrie dites géometiques, Paris 1934, S. $86 \mathrm{f}$.

72 Das „virtuelle Bild“ des Planspiegels, das in die zweite und dritte Version der Anordnung eingeführt wird, liegt hinter der Spiegelebene.

73 Vgl. Lacan 1953-54/1990: 181.

74 Pelwijk/Tinbergen 1937: 194.

75 Vgl. Kortlandt 1940, Tinbergen 1940 und 1946.

76 Vgl. Tinbergen 1940: 12 ff.; und Kortlandt 1940.

77 Vgl. Tinbergen 1940: 2.

78 Tinbergen 1940: 13.

79 Tinbergen 1940.

80 Lacan 1953/2002: 22.

81 „Ein wahrhafter Tanz, eine Art Hochzeitsflug findet statt, bei dem es zunächst darum geht, das Weibchen zu bezaubern, dann es sanft dazu zu verleiten, sich es sich gefallen zu lassen (à se laisser faire) und in einer Art kleinem Tunnel zu nisten, das man ihm zuvor verfertigt hat." (Lacan 1955-56/1997: 113).

82 Lacan 1955-56/1997: 113.

83 Es scheint der Rhetorik geschuldet, daß die kleinen Löcher während des Sexualzyklus fabriziert werden: denn das Argument läuft ja grade darauf zu, daß in den Kampfzyklus ein Fragment des Sexualzyklus, alias Nestbau, eingeschoben wird.

84 Über ein ähnliches Verfahren Lacans im Seminar über die vier Grundbegriffe vgl. Peter Berz, Die vier Verschiebungen des Blicks, in: Blümle/von der Heiden 2005, S. 183-216.

85 Sartre 1943/1993: 1046.

86 Sartre 1943/1993: 1048. - Daß Sartres Psychologie des menschlichen Wesens „,beim Bourgeois“ landet, „,der dabei ist, sich am Strand zu zerstreuen“, ist freilich eine Urlaubsphantasie Lacans (Lacan 1955-56/1997: 113) .

87 Vgl. jüngst Julian Furrer Topologie als Seinsgeschichte. Heidegger und die Löcher (Magisterarbeit im Fach Kulturwissenschaft, Berlin 2011). Darin auch der Hin- 
weis auf die weder Sartre noch Lacan und die Stichlinge bedenkende Psychoanalyse der Löcher bei Ricardo Rodulfo (vgl. Ders., Kinder - Gibt es die?. Die lange Geburt des Subjekts, Wien 1995, S. 123-140, „Über das Loch“).

88 Lacan 1955-56/1997: 113.

89 Sartre 1943/1993: 114.

90 Kortlandt 1940: $476 \mathrm{f}$.

91 Lacan 1953/2002: 20. - „Sagen wir, daß in der Tierwelt der ganze Zyklus des sexuellen Verhaltens vom Imaginären beherrscht ist." (Lacan 1953-54/1990: 177).

92 Lacan 1953-54/1990: 177.

93 Lacan 1953/2002: 23.

94 Lacan 1953/2002: 23.

95 Mit Parade, Verführung, Despotismus läßt Lacan schon 1938 im EnzyklopedieArtikel über „Die Familie“ früheste Identifikationsformen im Spiel zweier etwa gleich-altriger Kinder im Alter von einem halben bis zu zwei Jahren beginnen (vgl. Lacan 1938/1980: 55 und 60).

96 Daß Lacan ganz selbstverständlich vom „tierischen Subjekt“ spricht, würde am Ende ebenso von seiner lamarckistischen Inspiration zeugen wie davon, daß das lacansche Subjekt eben nichts mit Dem Menschen zu tun hat.

97 Vgl. etwa Tinbergen 1948: 10.

98 Lacan 1955-56/1997: 114.

99 Ulrike Kadi spricht genau an dieser Stelle, mit Verweis auf Portmann, von Lacans „ungenauem Begriff von Natur“ (Kadi 1999: 70) und beruft sich auch auf das Diktum von einer ,heimlich-verbotenen Verbindung von transzendentaler Philosophie und empirischer Biologie" in Lacans frühem Denken (Hanna Gekle 1996, zit. bei Kadi 1999: 214, Anm 14). Eine Analyse der vielfältigen, wenig heimlichen und diskursstrategisch sehr reflektierten biologischen Elemente in Lacans Diskursanordnungen könnte am Ende ein anderes Licht auf diese Fragen werfen.

100 Portmann 1944/1951: 47.

101 Portmann 1944/1951: 27 und 31.

102 Portmann 1944/1951: 30. - Zu Bühlers Begriff des Spielraums vgl. weiter oben.

103 Portmann 1944/1951: 47.

104 Portmann 1944/1951: 47 und 68. - Zwei wichtige Beweise für den „fötalen Charakter der Frühzeit": bei allen höheren Säugetieren sind die Neugeborenen „,verkleinerte Abbilder der Reifeform“, beim Menschen nach allen Proportionen nur entfernt, jedenfalls, wenn man sich nicht, wie üblich, allein auf Kopf und 
Gesicht fixiert; im ersten Jahr wächst das Menschenkind sehr viel schneller als alle anderen Säugetiere, nach dem ersten Jahr aber um ein Vielfaches langsamer (vgl. Portmann 1944/1951: 33-37 und 46 f.).

105 Vgl. Portmann 1944/1951: 51 und 77 ff.

106 Portmann 1944/1951: 51 und 77 ff. - Damit relativiere sich, so Portmann, auch die Reichweite von Haeckels ,biogenetischem Grundgesetz“.

107 Vgl. Portmann 1944/1951: 70-71, 74-76.

108 Portmann 1944/1951: 78.

109 Portmann 1944/1951: 79.

110 Vgl. etwa Portmann 1944/1951: 78.

111 Lacan 1953-54/1990: $217 \mathrm{f}$.

112 So taucht etwa das „Körperschema“, ein Konzept des Wiener Psychiaters und Kriegsversehrtenarztes Paul Schilder (1923), in der Phänomenologie Schelers und Merleau-Pontys auf. In der französischen Psychoanalyse denken vor allem Henri Wallon (1931 und 1934) und Jean Lhermitte (1939) über la notion, l'image du corps propre nach.

113 Vgl. Lacan 1936-1949/1973: 65.

114 Vgl. Lacan 1936-1949/1973: 66.

115 Lacan 1955-56/1997: 18. - „Das ist übrigens auch die einzige wirklich fundamentale Differenz zwischen der menschlichen Psychologie und der tierischen Psychologie. Der Mensch weiß sich als Körper ..." (Lacan 1953-54/1990: 218).

116 Lacan 1953-54/1990: 218.

117 Später im Seminar wird die Dialektik der Anerkennung in Hegels Herr und Knecht explizit zum Thema (vgl. Lacan 1953-54/1990: 281-283).

118 Lacan 1955-56/1997: 115.

119 Vgl. Walter 1953/1961: 127-145 (mit Dank an Jan Müggenburg, Wien/Lüneburg!). Vgl. auch Lacan 1954-55/1991: 74.

120 Walter 1953/1961: 141.

121 Lacan 1955-56/1997: 115.

122 Lacan 1953-54/1990: 219.

123 Lacan 1954-55/1991: 44.

124 Lacan 1955-56/1997: 116.

125 Lacan 1954-55/1991: 99. 


\section{Literatur}

Blümle, Claudia und von der Heiden, Anne (Hg.), 2005: Blickzähmung und Augentäuschung. Zu Facques Lacans Bildtheorie, Zürich, Berlin.

Bühler, Karl, 1929: Die Krise der Psychologie, Jena.

Caillois, Roger, 1935/2007: „Mimese und legendäre Psychasthenie“ (1935), in: Ders., Méduse Ė Cie., Berlin, S. 24-43.

Dalí, Salvador, 1933/1974: „Paranoisch-kritische Interpretation des zwanghaften Bildes ,Das Abendläuten“ von Millet“, in: Ders., Unabhängigkeitserklärung und Erklärung der Rechte des Menschen auf seine Verrücktheit. Gesammelte Schriften, hg. Axel Matthes und Tilbert Diego Stegmann, Frankfurt a. M. 1974, S. 196 - 202 (frz.: Minotaure, no. 1, 1933).

Foucault, Michel, 1961/1973: Wahnsinn und Gesellschaft. Eine Geschichte des Wahns im Zeitalter der Vernunft (übersetzt von Ulrich Köppen), Frankfurt a. M. (frz.: Histoire de la folie, Paris 1961).

Francé-Harrar, Annie, 1926: Tier und Liebe. Geschichten von Unterdrückten und Verkannten, Berlin.

Freud, Sigmund, 1895/1950: Entwurf einer Psychologie (1895), in: Ders., Aus den Anfängen der Psychoanalyse. Briefe an Wilhelm Fließ, Abhandlungen und Notizen aus den Fahren 18871902, hg. Marie Bonaparte, Anna Freud, Ernst Kris, Frankfurt a. M., S. 297-384.

Haraway, Donna F., 2008: When species meet (Posthumanities vol. 3), University of Minnesota Press.

Hegel, Gottfried-Wilhelm Friedrich, 1830/1970: Enzyklopädie der philosophischen Wissenschaften im Grundrisse, Zweiter Teil. Die Naturphilosophie. Mit mündlichen Zusätzen, in: Ders., Werke, Band 9, Frankfurt a. M..

Heidegger, Martin, 1935/1987: Die Frage nach dem Ding. Zu Kants Lehre von den transzendentalen Grundsätzen (Vorlesung 1935/36), Tübingen.

Hofer, Veronika, „Konrad Lorenz als Schüler von Karl Bühler. Diskussion der neu entdeckten Quellen zu den persönlichen und inhaltlichen Positionen zwischen Karl Bühler, Korad Lorenz und Egon Brunswick", in: Zeitgeschichte 3, 28. Jahrgang, 2002, S. 135-159.

Kadi, Ulrike, 1999: Bilderwahn. Arbeit am Imaginären, Wien.

Kortlandt, Adriaan, 1940: „Wechselwirkung zwischen Instinkten“, in: Archives Néerlandaises de Zoologie, t. IV, 4e livr., nov. 1940, S. 443-520.

Lacan, Jacques, 1932/2002: „Über die paranoische Psychose in ihren Beziehungen zur Persönlichkeit" (1932), in: ders., Über die paranoische Psychose in ihren Beziehungen zur Persönlichkeit und Frühe Schriften über die Paranoia, übersetzt von Hans- 
Dieter Gondek, Wien 2002.

- 1936-1949/1973: „Das Spiegelstadium als Bildner der Ichfunktion wie sie uns in der psychoanalytischen Erfahrung erscheint. Bericht für den 16. Internationalen Kongreß für Psychoanalyse in Zürich am 17. Juli 1949“ (übersetzt von Peter Stehlin), in: ders., Schriften I, Frankfurt a. M. 1975, S. 61-70.

- 1936/1994: ,Jenseits des Realitätsprinzips“ (übersetzt von Franz Kaltenbeck), in: ders., Schriften III, Olten, S. 17-37.

- 1938/1980: „Die Familie“ (1938), übersetzt von Friedrich Kittler, in: ders., Schriften III, Olten 1980, S. 39-100.

- 1953/2006: „Das Symbolische, das Imaginäre und das Reale (8. Juli 1953)“, in: ders., Namen-des-Vaters, übersetzt von Hans-Dieter Gondek, Wien, S. 11-61.

- 1953-54/1990: Freuds technische Schriften. Das Seminar Buch I (1953-1954), übersetzt von Werner Hamacher, Weinheim und Berlin (frz. 1975).

- 1954-55/1991: Das Ich in der Theorie Freuds und in der Psychoanalyse. Das Seminar Buch II (1954 -1955), übersetzt von Hans-Joachim Metzger, Weinheim und Berlin (frz. 1978).

- 1955-56/1997: Die Psychosen. Das Seminar Buch III (1955-1956), übersetzt von Micheal Thurnheim, Weinheim und Berlin (frz. 1981).

- 1966/1980: „Von dem, was uns vorausging“ (übersetzt von Norbert Haas), in: ders., Schriften III, Olten, S. 9-14.

Lorenz, Konrad, 1935: „Der Kumpan in der Umwelt des Vogels. Der Artgenosse als auslösendes Moment sozialer Verhaltungsweisen. Jakob von Uexküll zum 70. Geburtstag gewidmet“, in: Journal für Ornithologie, 83. Jg., Heft 2, April 1935, S. 137-413.

- 1949/1970: Er redete mit dem Vieh, den Vögeln und den Fischen (1949), 15. Auflage, München.

- 1949/1953: Les animaux, ces inconnus, traduit par Ct. Jouan, Paris (Übersetzung von: Er redete mit dem Vieh, den Vögeln und den Fischen, 1949).

- 1950/1965: „Ganzheit und Teil in der tierischen und menschlichen Gemeinschaft. Eine methodolgische Erörterung“ (1950), in: ders., Über tierisches und menschliches Verhalten. Aus dem Werdegang der Verhaltenslehre. Gesammelte Abhandlungen Band I und II, S. 422-491.

McFarland, David, 1985/1989: Biologie des Verhaltens. Evolution, Physiologie, Psychobiologie, Weinheim (engl. 1985).

Portmann, Adolf, 1944/1951: Biologische Fragmente zu einer Lehre vom Menschen, zweite, erweiterte Auflage, Basel.

- 1948/1965: Die Tiergestalt. Studien über die Bedeutung der tierischen Erscheinung, zweite Auflage, Freiburg Basel Wien.

— 1950/1956: „Das Problem der Urbilder in biologischer Sicht“, in: ders., Biologie 
und Geist, Zürich, S. 133-149.

Sartre, Jean-Paul, 1943/1993: Das Sein und das Nichts. Versuch einer phänomenologischen Ontologie, übersetzt von Hans Schöneberg und Traugott König, Hamburg.

Ter Pelkwijk, J. J., und Tinbergen, Nikolaas, 1937: „Eine reizbiologische Analyse einiger Verhaltensweisen von Gasterosteus aculeatus L.", in: Zeitschrift für Tierpsychologie, 1. Band, 1937, S. 193-200.

Tinbergen, Nikolaas, 1940: „Die Übersprungsbewegung“, in: Zeitschrift für Tierpsychologie, 4. Band, 1940/41, S. 1-40.

- 1946: „,Displacement reactions“ in the three-spined stickleback“, in: Behaviour. An international journal of comparative ethology, vol. 1, part 1, S. 56-63.

- 1948: „Social releasers and the experimental method required for their study“, in: The Wilson Bulletin, vol. 60, No. 1, march 1948, S. 6-51.

- 1952: Instinktlehre. Vergleichende Erforschung angeborenen Verhaltens, Berlin und Hamburg (engl. 1950).

Uexküll, Jakob von, 1909/1921: Umwelt und Innenwelt der Tiere, 2. Auflage, Berlin.

Walter, William Grey, 1953/1961: Das lebende Gehirn. Entwicklung und Funktion (übertragen von Gertrud Walther), Köln Berlin.

\section{Bildnachweise}

Abb. 1: Brehms Tierleben. Allgemeine Kunde des Tierreichs, 4., vollständig neu bearbeitete Auflage (hg. Otto zu Strassen), Leipzig und Wien, 1914, Band: Fische, S. 353.

Abb. 2: Urania Tierreich in sechs Bänden, Jena, Berlin 1967, Band: Fische Lurche Kriechtiere, S. 222.

Abb. 3: J. J. Ter Pelkwijk, N. Tinbergen, „Eine reizbiologische Analyse einiger Verhaltensweisen von Gasterosteus aculeatus L.“, in: Zeitschrift für Tierpsychologie, 1. Bd., 1937, S. 193-200, 197.

Abb. 4: J. J. Ter Pelkwijk, N. Tinbergen, „Eine reizbiologische Analyse einiger Verhaltensweisen von Gasterosteus aculeatus L.“, in: Zeitschrift für Tierpsychologie, 1. Bd., 1937, S. 193-200, 194.

Abb. 5: Nicolaas Tinbergen, Instinktlehre. Vergleichende Erforschung angeborenen Verhaltens (übersetzt von O. Köhler), Berlin und Hamburg, 1952 (engl. 1950), Frontispiz.

Abb. 6: Nicolaas Tinbergen, „Die Übersprungsbewegung“, in: Zeitschrift für Tierpsychologie, 4. Bd., 1940/41, S. 1-40, 14.

Abb. 7: Nicolaas Tinbergen, Instinktlehre. Vergleichende Erforschung angeborenen Verhaltens (übersetzt von O. Köhler), Berlin und Hamburg, 1952 (engl. 1950), S. 110. 